\title{
Acute bilateral thalamic infarctions with midbrain involvement due to artery of Percheron occlusion
}

\author{
Nadeeshani PGN ${ }^{1}$, Liyanage $A^{1}$, Rajendiran $\mathbf{T}^{1}$, Indrakumar $\mathbf{J}^{2}$ \\ Journal of the Ceylon College of Physicians, 2020, 51, 136-138
}

\begin{abstract}
The artery of Percheron is a single dominant vessel of posterior cerebral artery which supplies the thalamus bilaterally. It is a rare variant of arterial supply to the paramedian thalami. The classical triad of presentation of artery of Percheron occlusion is vertical gaze palsy, memory impairment, and alteration of mental status. Here we report a case of artery of Percheron occlusion where the patient presents with classic features of thalamic infarction associated with the midbrain involvement evidenced by the presence of bilateral hemiparesis. When compared to the typical thalamic infarction alone, the prognosis is worse when midbrain is also affected
\end{abstract}

Key words: bilateral thalamic infarctions, artery of Percheron occlusion, midbrain

\section{Introduction}

The artery of Percheron is an uncommon anatomical variant of arterial supply to the paramedian thalamus and rostral midbrain. Occlusion of the artery of Percheron results in bilateral paramedian thalamic infarcts with or without midbrain involvement. Acute infarctions due to artery of Percheron occlusion represents 0.1 to $2 \%$ of all ischemic strokes.

\section{Case report}

A 54-year-old woman, with a history of diabetes taking metformin and gliclazide, was admitted with sudden onset of reduced responsiveness. She was in good health until two days before when she had developed diarrhea and vomiting. She was treated with rehydration solutions and domperidone by the family physician. There was no significant past medical history other than diabetes and she did not smoke, take alcohol or illicit drugs.

On admission to the hospital, she was deeply somnolent. Her blood pressure was $130 / 80 \mathrm{mmHg}$, pulse rate was $80 \mathrm{bpm}$, and oxygen saturation was $100 \%$. She was afebrile and had no neck stiffness. Random blood glucose was $154 \mathrm{mg} / \mathrm{dl}$. There was complete ptosis of the right eye and paresis of adduction, upward and downward gaze. The right-side pupil was dilated with impaired light reflexes. Examination of both upper and lower limbs showed bilateral asymmetrical (right>left and upper limb > lower limb) weakness.

Her investigations showed white cell count 5.05 $\times 10^{9} \mathrm{~L}$, haemoglobin $12 \mathrm{~g} / \mathrm{dL}$, platelets $232 \times 10^{9} \mathrm{~L}$, serum sodium $138 \mathrm{mmol} / \mathrm{l}$, serum potassium $4.7 \mathrm{mmol} / \mathrm{l}, \mathrm{CRP}<5 \mathrm{mg} / \mathrm{L}$, and serum creatinine 83 micrmols/L. Non-contrast computed tomography (CT) brain showed features suggestive of bilateral thalamic infarcts (Figure 1). Magnetic resonance imaging (MRI) of the brain done two days later confirmed bilateral subacute thalamic and midbrain infarcts compatible with occlusion of the artery of Percheron. A 2D echocardiography and bilateral carotid duplex scan did not show any source of thromboembolism.

\footnotetext{
${ }^{1}$ Colombo South Teaching Hospital, Sri Lanka, ${ }^{2}$ Faculty of Medical Sciences, University of Sri Jayewardenepura, Sri Lanka

Correspondence:PGNN, e-mail:nelum_pg@yahoo.com

D https://orcid.org/0000-0002-1052-704

Received 18 Febuary 2020, accepted 20 May 2020.

This is an open-access article distributed under the terms of the Creative Commons Attribution License, which permits unrestricted use, distribution, and reproduction in any medium, provided the original author and source are credited.
} 


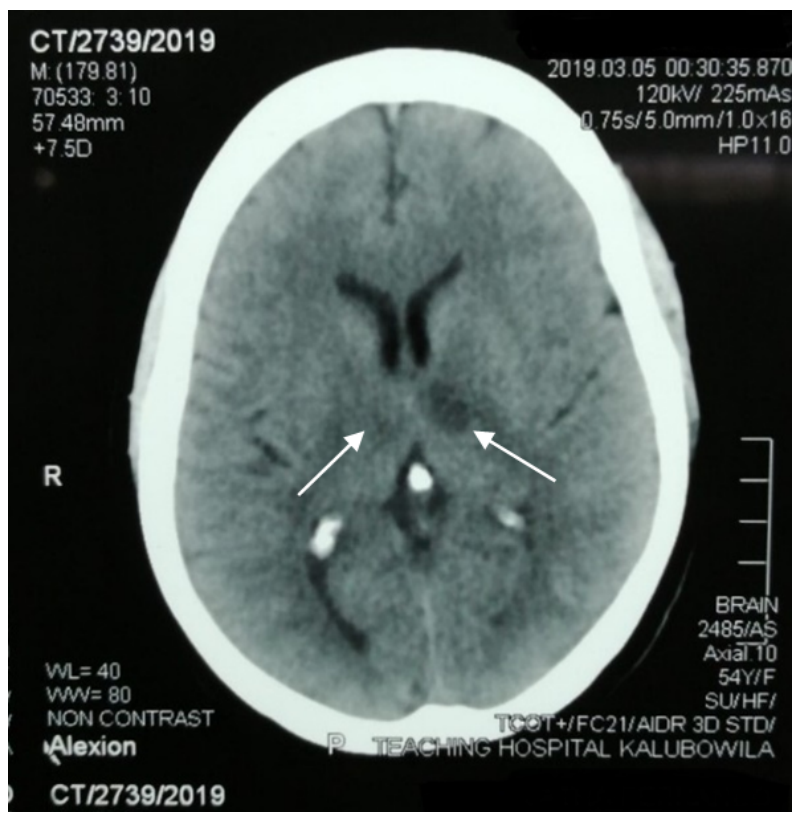

Figure 1. NCCT Brain with bilateral thalamic infarctions (white arrows).

She was treated with aspirin $75 \mathrm{mg}$ and atorvastatin $20 \mathrm{mg}$ daily. Her consciousness started to improve gradually from day 3 of admission with intermittent periods of somnolence. She showed difficulty recalling her personal history and social events that occurred in the past ten years. After 2 weeks in the hospital, she was conscious with a GCS of 15/15. Subsequently, she was discharged from the hospital and followed up at medical clinic.

\section{Discussion}

The blood supply of the thalamus is predominantly from the perforating branches of the posterior cerebral artery. ${ }^{1}$ There are 4 major thalamic vascular territories, namely the tuberothalamic, inferolateral, paramedian, and posterior choroidal area, each with an arterial supply by multiple perforating vessels. ${ }^{2}$ There is a wide variability of arterial supply among individuals (Figure 2). Here we describe one such important uncommon variant artery leading to a clinical syndrome called 'Artery of Percheron'. It is named after the French neurologist Gérard Percheron, who described it in 1973. ${ }^{1}$

The artery of Percheron is a part of the posterior cerebral circulation characterized by a solitary artery that supplies blood to the bilateral paramedian thalami and the rostral midbrain. ${ }^{2,3}$ As compared to multiple bilateral perforating vessels, occlusion of this single arterial trunk can easily lead to significant midbrain and/ or thalamic damage. ${ }^{3}$

The artery of Percheron is estimated to be present in up to $33 \%$ of the population. ${ }^{3}$ About $0.1-2 \%$ of all ischaemic strokes involve the artery of Percheron. ${ }^{1}$ Strokes limited to paramedian territories account for about $22 \%$ to $35 \%$ of all thalamic infarcts. ${ }^{4}$ The incidence of bilateral infarcts limited to this vascular territory is of $4-18 \%$ of all thalamic strokes. ${ }^{4}$ The classic triad of presentation of this condition is ophthalmologic signs (vertical gaze palsy and/or abnormal pupillary reflex), memory impairment and altered mental status. ${ }^{1}$ Patients often present with altered mental status or any type of amnesia due to thalamic involvement. ${ }^{5}$ In the acute setting, these symptoms may mimic conditions like encephalitis, metabolic or toxic encephalopathies. ${ }^{5}$ In most cases, cognitive and behavioral changes become obvious only when consciousness resumes. ${ }^{5}$ In some instances, it is associated with gaze palsies, hemisensory loss, hemiparesis and cerebellar defects secondary to midbrain involvement. ${ }^{5}$
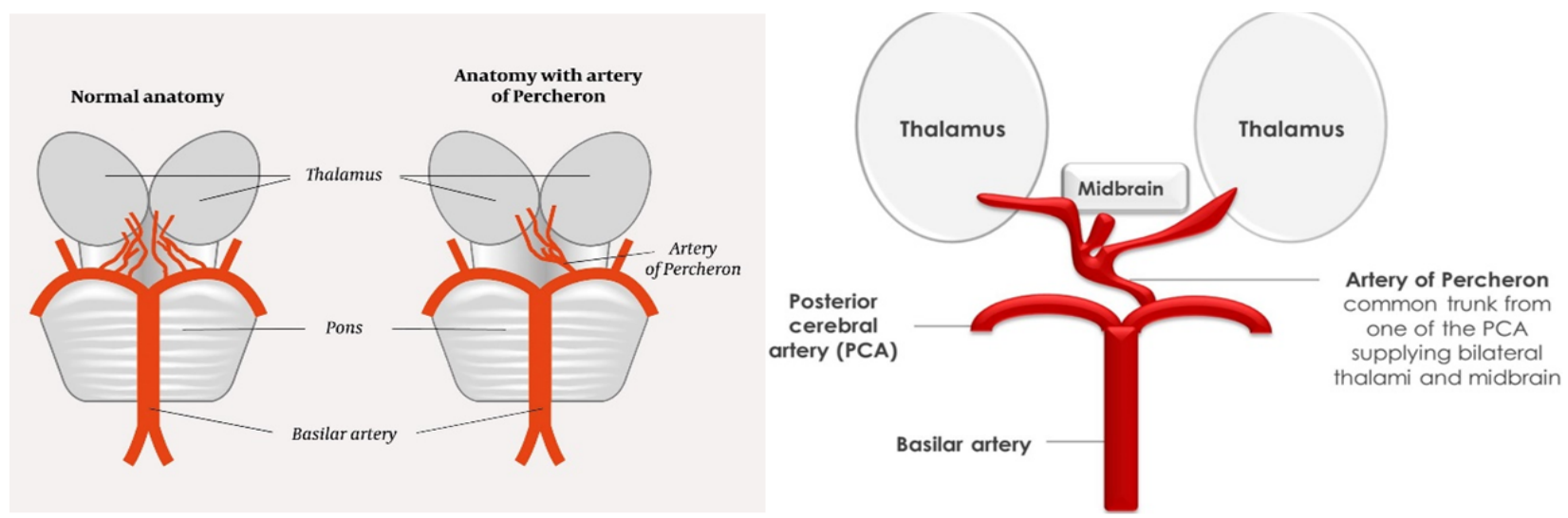

Figure 2. Anatomy of the variant artery - artery of Percheron supplying both thalami and midbrain. 


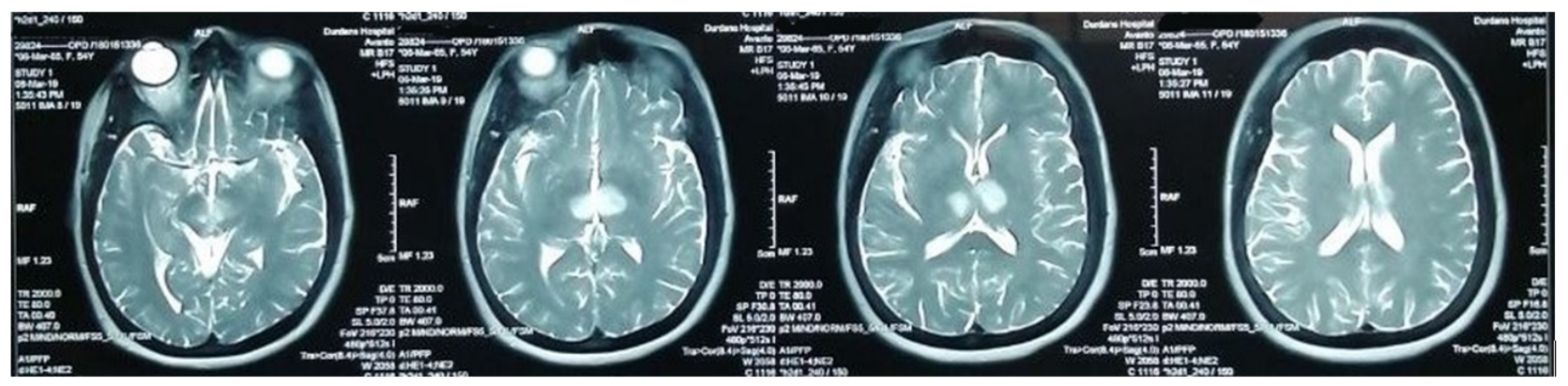

Figure 3. MRI Brain with thalamic and midbrain infarction.

Our patient presented with not only the classic bilateral thalamic involvement but also an uncommon additional midbrain involvement compatible with the artery of Percheron occlusion. Vertical gaze palsy is characteristic of bilateral thalamic infarctions. In our patient bilateral paramedian midbrain infarction caused the weakness of limbs and ophthalmic involvement due to nuclear and fascicular involvement. Detailed cognitive and memory assessment done after recovery of conscious state showed that she had predominant recent memory loss but was able to read and understand.

In acute stroke, due to the artery of Percheron occlusion, early brain non-contrast CT is usually normal. Therefore, MRI is the gold standard imaging modality. ${ }^{6}$ Classical MRI findings of high signal on fluid-attenuated inversion recovery (FLAIR) and diffusion-weighted imaging (T2) with the corresponding restriction on apparent diffusion coefficient in paramedian nuclei were present in our patient along with the additional involvement in rostral midbrain ${ }^{6}$ (Figure 3).

Emergency treatment of this condition is the reperfusion of the ischaemic brain tissues. Intravenous thrombolysis with tissue plasminogen activator is the mainstay of initial treatment as for other ischaemic strokes if the patient presents within the thrombolytic time frame. ${ }^{7}$ No consensus currently exists on the role of mechanical thrombectomy. ${ }^{1}$ Long-term antiplatelets, statins, and anticoagulants where appropriate are used to prevent further ischaemic episodes. ${ }^{1}$

Artery of Percheron infarction has a favorable long term prognosis. ${ }^{5}$ However, the prognosis is said to be worse in the presence of additional midbrain infarction when compared to isolated thalamic infarcts as in this case. ${ }^{8}$ This is due to the persistence of cognitive and psychiatric manifestations with associated motor deficits. ${ }^{8}$ Contrary to this prediction, our patient improved markedly over the next 4 months with regard to her motor weakness and with only residual mild memory deficits which are progressively improving.

\section{Conclusion}

Bilateral thalamic infarction due to artery of Percheron involvement is a rare stroke syndrome. Although it usually manifests as thalamic infarctions, rarely it can involve the midbrain. Although it is known that when both thalamus and midbrain are involved prognosis is worse, our patient demonstrated a remarkable improvement in motor weakness and memory impairment.

\section{Acknowledgment}

Dr. Apsara Epa, Consultant Radiologist, Colombo South Teaching Hospital, Sri Lanka.

\section{References}

1. Ahmed A, Stuart M, Snehal L. Case Report; Artery of Percheron infarction. British Journal of Hospital Medicine 2019; 80: 412-13.

2. Jeremy D. Schmahmann. Vascular Syndromes of the Thalamus. Stroke 2003; 9: 2264-78.

3. Percheron $\mathrm{G}$. The anatomy of the arterial supply of the human thalamus and its use for the interpretation of the thalamic vascular pathology. Neurolol. 1973; 205(1): 1-13.

4. López-Serna R, González-Carmona P, López-Martínez M. Bilateral thalamic stroke due to occlusion of the artery of Percheron in a patient with patent foramen ovale: a case report. J Med Case Reports. 2009; 3: 7392 https://dx.doi.org/ 10.4076\%2F1752-1947-3-7392

5. DeSilva CM, Fonseka CL, Singhapura SDAL, Kularatne OAR, Jayasekara JMS, Rajapaksha H. The Lady who underwent twenty years back in her life; a case of acute bilateral thalamic infarction due to artery of Percheron involvement. JCCP 2017; 48: 85-7.

6. Matheus MG, Castillo M. Imaging of acute bilateral paramedian thalamic and mesencephalic infarcts. Am J Neuroradiol 2003; 24(10): 2005-8.

7. Li X, Agarwal N, Hansberry DR, Prestigiacomo CJ, Gandhi CD. Contemporary therapeutic strategies for occlusion of the artery of Percheron: a review of the literature. Journal of Neurointerventional Surgery 2015; 7(2): 95-8.

8. Kostanian V, Cramer S.C. Artery of Percheron thrombolysis. Am J Neuroradiol. 2007; 28; 870-1. 